\title{
A Clonorchiasis Case of a Leopard Cat, Prionailurus bengalensis euptilurus, Diagnosed by Ultrasonography and Egg Detection in Republic of Korea
}

\author{
Seongjun Choe' ${ }^{1}$, Dong-Hyuk Jeong ${ }^{2}$, Jeong-Jin Yang ${ }^{2}$, Jeongho Kim³ ${ }^{3}$ Ki-Jeong $\mathrm{Na}^{4,5}$, Dongmin Lee', \\ Hansol Park', Hyeong-Kyu Jeon ${ }^{1}$, Keeseon S. Eom ${ }^{1, *}$ \\ 'Department of Parasitology, Parasite Research Center and Parasite Resource Bank, School of Medicine, Chungbuk National University, Cheongju \\ 28644, Korea; '2Species Restoration Technology Institute, Korea National Park Service, Gurye 57616, Korea; ${ }^{3}$ Cheongju Zoo, Cheongju 28311 , \\ Korea; ${ }^{4}$ The Wildlife Center of Chungbuk, Cheongju 28116, Korea; ${ }^{5}$ College of Veterinary Medicine, Chungbuk National University, Cheongju 28644, \\ Korea
}

\begin{abstract}
A clonorchiasis case in a captive leopard cat, Prionailurus bengalensis euptilurus, was confirmed by ultrasonographic findings and egg morphologies found in the bile juice sample in the Korea. The leopard cat was introduced from the wild habitat of Gyeongsangnam-do, to Cheongju Zoo in Cheongju-si, Chungcheongbuk-do, Korea in August 2014. Physical examinations were basically performed for quarantine and check-up health. The cat was comparatively good in health except anorexia. The cyst-like bile duct dilation and the increased echogenicity of gall bladder wall and hepatic parenchyma were observed by ultrasonography. Ultrasound-guided needle biopsy was conducted for collecting bile juice and the specimens were observed under light microscope. The numerous small trematode eggs were detected in the bile juice sample of the light microscopy. The eggs were 25-33 (28 \pm 3$) \mu \mathrm{m}$ by 18-22 $(20 \pm 1) \mu \mathrm{m}$ in size and showed typical characteristics of Clonorchis sinensis egg, i.e., a dominantly developed operculum, shoulder rim and dust-like wrinkles in surface. To treat the liver fluke infection, $20 \mathrm{mg} / \mathrm{kg}$ of praziquantel was orally administered only once to the case. Follow-up studies including fecal examinations were conducted during 2 years after treatment. But no more eggs were detected from the case. In the present study, we described the first clonorchiasis case of leopard cat, which was confirmed by ultrasonographic findings and egg morphologies from the bile juice sample in Korea.
\end{abstract}

Key words: Clonorchiasis, Prionailurus bengalensis euptilurus, ultrasonography

\section{INTRODUCTION}

Clonorchiasis is an infectious disease occurs in the Far East countries including the Republic of Korea (Korea), China, Japan, Taiwan, northern Vietnam and the far eastern part of Russia [1]. This disease is caused by opisthorchiid liver fluke, Clonorchis sinensis, and it infected in biliary system of mammalian hosts including human who eating raw fishes [1]. It has been known that most case of clonorchiasis is asymptomatic but, the heavily infected case sometimes manifests several symptoms such as cholangitis, biliary obstruction, dilation and wall thickening of bile duct, liver cirrhosis, and even develops chol-

\footnotetext{
- Received 10 January 2019, revised 8 April 2019, accepted 10 April 2019

*Corresponding author (kseom@chungbuk.ac.kr)

(c) 2019, Korean Society for Parasitology and Tropical Medicine

This is an Open Access article distributed under the terms of the Creative Commons Attribution Non-Commercial License (http://creativecommons.org/licenses/by-nc/4.0) which permits unrestricted non-commercial use, distribution, and reproduction in any medium, provided the original work is properly cited.
}

angiocarcinoma in human [1,2].

In human, ultrasonography is one of the diagnostic methods used for clonorchiasis. This diagnostic device is able to detect the characteristic sonographic findings, i.e., increased periductal echogenicity and floating echogenic foci in gall bladder, due to the infections of liver flukes. The positive findings by ultrasonography strongly suggest the active clonorchiasis [3]. Ultrasonography provides only indirect evidence of clonorchiasis and is not easy to detect in light or early infection cases $[3,4]$. But it is considered as a useful and simple technique for detecting pathological changes in biliary system due to the disease, especially in patients with a moderate to heavy infection $[1,5]$.

In other animals, ultrasonography was poorly studied as a diagnostic tool for clonorchiasis. Although several studies were conducted for other liver fluke species, Platynosomum fatosum and Opisthorcis viverrini in cat $[6,7]$, but it is not been conducted for C. sinensis until the present study. Purpose of the present 
study is to describe a case of clonorchiasis in leopard cat, Prionailurus bengalensis euptilurus which accidentally detected during physical examination using ultrasonography and bile juice suction drainage study.

\section{CASE DESCRIPTION}

A leopard cat was introduced from Gyeongsangnam-do Forest Environment Research Institute, Jinju-si (city), Gyeongsangnam-do (province) to Cheongju Zoo, in Cheongju-si, Chungcheongbuk-do, Korea in August 2014. The cat was adult female, $3.26 \mathrm{~kg}$ in weight. It was known as a captive animal which was rescued from wild habitat in Gyeongsangnam-do but the exact locality was unknown. During the introduction process, chicken meat was given daily and mice were fed in a week but, no fishes were given. Vaccines and parasiticides had not been administered. For quarantine and health check-up, zolazepam-tiletamine (Zoletile 50, Virbac Laboratories, Carros, France) $10 \mathrm{mg} / \mathrm{kg}$ and medetomidine (Domitor; Zoetis, Florham Park, New Jersey) $0.1 \mathrm{mg} / \mathrm{kg}$ were intramuscularly administrated and blood samples were collected from jugular vein. Then, general physical examination was performed, and blood chemistry was analyzed. The body condition of cat was comparatively good and not shown any typical symptoms with normal blood chemistry values except anorexia. Fecal examination using direct smear method resulted as negative for parasites. Additionally, abdominal ultrasonography was conducted because of the anorexia. At the ultrasonography imaging, cyst-like bile duct dilatation was observed nearly gall bladder. Echogenicity of walls of gall bladder and dilated bile duct were seemed slightly increased, and floating or deposited

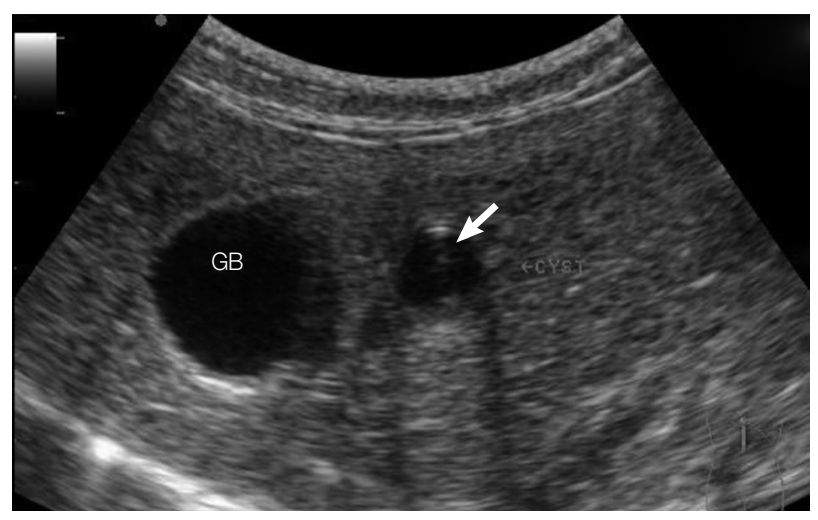

Fig. 1. Ultrasonogram on the liver of $C$. sinensis-infected leopard cat shows gall bladder (GB) and cyst-like bile duct dilation. Floating or sedimented echogenetic foci (arrow) were detected in a cyst. echogenic foci were detected from there.

Ultrasound-guided needle biopsy was performed for diagnosis the bile duct dilatation and identity of the echogenic foci (Fig. 1). Few millimeters of Bile juice with the debris were collected and observed under light microscopy on the slide glass with cover slip. Numerous parasite eggs were observed (Fig. 2).

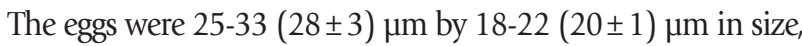
and they equipped with a dominantly developed small operculum, shoulder rim, dust-like wrinkles in surface and a miracidium inside. Observed parasite materials were deposited in the Parasite Resource Bank of Korea (PRB no. PRB001007). It was suggested that the cat infected with $C$. sinensis by the ultrasonographic findings, detection site of eggs and egg morphologies. To treat the liver fluke infection, $20 \mathrm{mg} / \mathrm{kg}$ of praziquantel (Distocide ${ }^{\circledR}$, Shinpoong Pharm. Co., Seoul, Korea) was orally administered only once to the case. Follow-up studies including regular fecal examinations were conducted during 2 years after the treatment. And no more liver fluke eggs were detected after the treatment, and she still maintained good health condition.

\section{DISCUSSION}

Korea is one of the endemic areas of clonorchiasis. Despite of successful parasite control for soil-transmitted helminthes [8], clonorchiasis has still remained with relatively high infection rate in human who living in endemic area near major riv-

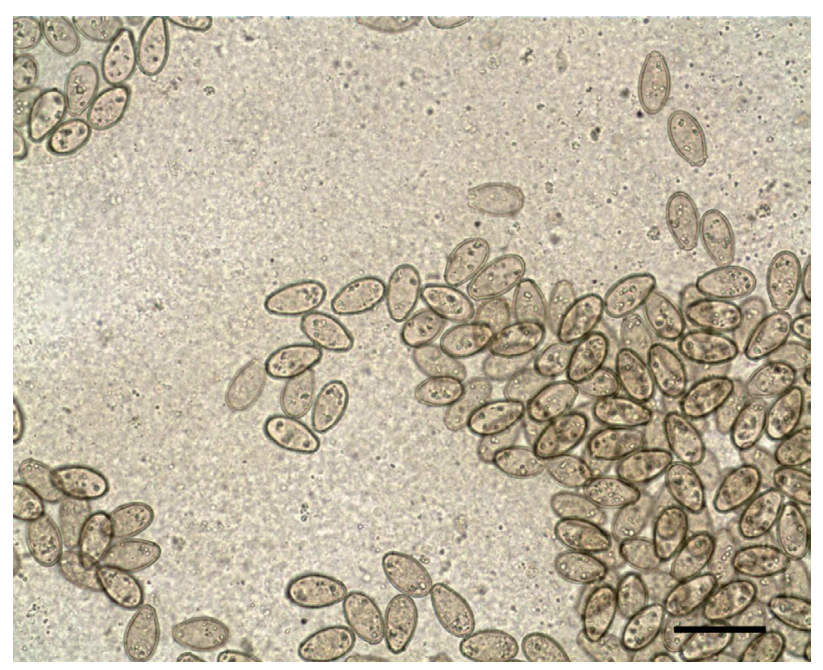

Fig. 2. Numerous $C$. sinensis eggs in a smear of bile juice drained from the leopard cat. Various characteristics like small operculum, shouldering and developed miracidium were partially observed in some of the eggs. Bar $=50 \mu \mathrm{m}$. 
er basins $[9,10]$. Recently, Jeong et al. [11] reported that the positive rate of helminth eggs is $9.5 \%(4,052)$ out of 42,562 fecal specimens of residents in the riverside areas of 5 major rivers in Korea. Among 4,052 helminth positive residents, 3,586 (88.5\%) ones are to be infected with C. sinensis. And then this finding suggested that clonorchiasis is the most important helminthic disease in Korea.

Compared with the concerning on human infections in Korea, the definitive host of $C$. sinensis are still required more study. Although lots of animals have been known to be infectable host of the fluke species, only dogs, cats and rats were reported as definitive hosts in Korea. For cats, 4 studies were found the infection cases of $C$. sinensis in Korea. Kang [12] found 3 cases of infected cats from a western region of Gyeongsangnam-do. Lee et al. [13] reported C. sinensis infection on cats from Gyeongsangbuk-do in 1979. And Huh et al. [14] investigated intestinal parasites of cats purchased at a market in Seoul. The positive ratio of Clonorchis infection on the cats was $12.2 \%$ (5/41). Later, Sohn and Chai investigated helminth parasites of cats purchased from a market in Busan [15]. They found 24 (5.5\%) infected cats among 438 feral cats. As our knowledge, the leopard cat, P. bengalensis euptilurus was not been reported as a host of $C$. sinensis in Korea. Thus, we newly added this felid species to the host list of $C$. sinensis, and it is the first case of $C$. sinensis infection on wildlife in Korea.

Leopard cat $P$. bengalensis euptilurus is a native felid species only survived in Korea. Like other felids which were disappeared from Korean Peninsula such as tiger, leopard and lynx, this small felid species also proficient hunter. And they are currently considered as a top predator in Korea ecosystem. Lee et al. [16] investigated the food habits of this small hunter from 4 provinces of Korea by fecal examination. However, they did not find any evidences of consuming fishes which is well known infection source of $C$. sinensis. Considering the facts that the patient only consumed chicken meat during its captive and introducing periods, the leopard cat should have infected by $C$. sinensis in the wild habitats. Present case might be an evidence of fish-eating habit of leopard cat in Korea.

The patient, leopard cat did not show typical clinical signs of clonorchiasis in the present study. Similar with our case, Aunpromma et al. [7] reported that most of infected cats did not show apparent clinical symptoms. Only few heavy infected cats showed the clinical signs of lethargy, diarrhea and jaundice. In addition, their clinical signs and haematological profiles were not much different with non-infected cats [7].
They suggested that $O$. viverrini does not induce prominent effects on a particular animal species like cat unless it is highly infected [7]. The fact might be applicable to clonorchiasis on leopard cat as well.

The main tools for detecting clonorchiasis in our case were both of ultrasonography and ulatrasound-guided needle biopsy of bile juice. In the ultrasonogram, several findings which like bile duct dilation, floating echogenic foci in gall bladder, increased echogenicity of gall bladder wall and hepatobiliary tract were detected. These findings are very similar with the signs which detected from active clonorchiasis in human by ultrasonography. Ultrasonogram conducted on heavy infected cats by $O$. viverrini and $P$. fatosum also showed quite similar results with humans and our case $[6,7]$. These feautres are known to reflect periductal fibrosis with worm body and exudates of liver flukes in biliary system [3-7]. These pathological changes are not easy to detect in light or early infection cases by ultrasonography $[3,4]$. Thus, the patient might be infected with $C$. sinensis a long while ago.

Ultrasonography could be a useful indirect diagnostic tool for primary detection of clonorchiasis. Even in our case ultrasonography acted as additional filter for the detection although eggs of $C$. sinensis was not found at fecal examination. Indisciplined inspectors could miss the infection of C. sinensis due to its small size of the fluke ova. In addition, we used direct smear method for primal fecal examination. It could be a reason of the negative result.

In fact, our study has some limitation on the species identification. Because we did not preserve the egg specimens, we could not conduct the DNA analysis. Although we identified the eggs based on morphological characteristics and geographical information, these factors could be regarded as indirect method for species identification. Therefore, it should be conducted by recovering adult worm or DNA analysis.

Conclusively, we described a clonorchiasis case in a captive leopard cat, P. bengalensis euptilurus diagnosed by ultrasonographic findings and egg morphologies found in the bile juice sample in the Republic of Korea. Ultrasonography was conducted for the first time in leopard cat for detecting liver fluke infection and showed quite similar results with human cases and other species of liver fluke infections on cats. The results would be an example that the combinations of ultrasonography and bilejuice suction are possible to use as a detecting tool of clonorchiasis in leopard cat and other animals as well. Furthermore, present case was a suspected case of wildlife that are 
naturally infected with $C$. sinensis in their wild habitat. Thus, additional surveys are needed for evaluating the roles of wildlife as a definitive hosts of $C$. sinensis in Korea for ecological and public health concern.

\section{ACKNOWLEDGEMENT}

This work was partially supported by the National Research Foundation of Korea (No. 2017R1D1A3B03035976).

\section{CONFLICT OF INTERESTS}

The authors declare that they have no conflict of interest with this article.

\section{REFERENCES}

1. Rim HJ. Clonorchiasis: an update. J Helminthol 2005; 79: 269281.

2. Hong ST, Fang Y. Clonorchis sinensis and clonorchiasis, an update. Parasitol Int 2012; 61: 17-24.

3. Choi D, Hong ST, Lim JH, Cho SY, Rim HJ, Ji Z, Yuan R, Wang S. Sonographic findings of active Clonorchis sinensis infection. J Clin Ultrasound 2004; 32: 17-23.

4. Hong ST, Yoon K, Lee M, Seo M, Choi MH, Sim JS, Choi BI, Yun CK, Lee SH. Control of clonorchiasis by repeated praziquantel treatment and low diagnostic efficacy of sonography. Korean J Parasitol 1998; 36: 249-254.

5. Lim JH, Ko YT, Lee DH, Kim SY. Clonorchiasis: Sonographic findings in 59 proved cases. Am J Roentgenol 1989; 152: 761764.

6. Salomão M, Souza-Dantas LM, Mendes-de-Almeida F, Branco
AS, Bastos OPM, Sterman F, Labarthe N. Ultrasonography in hepatobiliary evaluation of domestic cats (Felis catus, L., 1758) infected by Platynosomum Looss, 1907. Intern J Appl Res Vet Med 2005; 3: 271-279.

7. Aunpromma S, Tangkawattana P, Papirom P, Kanjampa P, Tesana S, Sripa B, Tangkawattana S. High prevalence of Opisthorchis viverrini infection in reservoir hosts in four districts of Khon Kaen Province, and opisthorchiasis endemic area of Thailand. Parasitol Int 2012; 61: 60-64.

8. Hong ST, Chai JY, Choi MH, Huh S, Rim HJ, Lee SH. A successful experience of soil-transmitted helminth control in the Republic of Korea. Korean J Parasitol 2006; 44: 177-185.

9. Shin EH, Guk SM, Kim HJ, Lee SH, Chai JY. Trends in parasitic diseases in the Republic of Korea. Trends Parasitol 2008; 24: 143-150.

10. Cho SH, Lee KY, Lee BC, Cho PY, Cheun HI, Hong ST, Sohn WM, Kim TS. Prevalence of clonorchiasis in southern endemic areas of Korea in 2006. Korean J Parasitol 2008; 46: 133-137.

11. Jeong YI, Shin HE, Lee SE, Cheun HI, Ju JW, Kim JY, Park MY, Cho SH. Prevalence of Clonorchis sinensis infection among residents along 5 major rivers in the Republic of Korea. Korean J Parasitol 2016; 54: 215-219.

12. Kang HJ. Studies on the parasitic helminths of the cats in western province of Kyung Sang Nam-do. Res Bull Chinju Agric Coll 1967; 6: 91-96 (in Korean).

13. Lee SH, Chai JY, Hong ST. Synopsis of Medical Parasitology. Seoul, Korea. Korea Medical Publishing. 1996, pp 6.

14. Huh S, Sohn WM, Chai JY. Intestinal parasites of cats purchased in Seoul. Korean J Parasitol 1993; 31: 371-373.

15. Sohn WM, Chai JY. Infection status with helminthes in feral cats purchased from a market in Busan, Republic of Korea. Korean J Parasitol 2005; 43: 93-100.

16. Lee O, Lee S, Nam DH, Lee HY. Food habits of the leopard cat (Prionailurus bengalensis euptilurus) in Korea. Mamm Study 2014; 39: 43-46. 